Eindruck meint. Auf der anderen Seite zeigt die Studie aber auch, dass eine Lysetherapie bei diesen Patienten sicher und vergleichsweise risikoarm ist. Insbesondere die intrakraniellen Blutungen sind deutlich niedriger als in den großen Zulassungsstudien der Thrombolyse und in den Schlaganfalldatenbanken. Kritisch anzumerken ist, dass der Beobachtungszeitraum (d.h. der Zeitpunkt der Klinikentlassung) sehr kurz gewählt worden ist. Außerdem wäre es hilfreich gewesen, wenn das Outcome der Patienten mit einem minor stroke und Thrombolyse verglichen worden wäre mit dem der Patienten mit minor stroke und ohne Thrombolyse. Bis heute wirkt ja nach, dass ursprünglich eine Thrombolyse formal nur bei einem Schlaganfall mit einem NIH-SS von über 4 zugelassen worden war.

Prof. Dr. med.Dr. phil. Stefan Evers

Romano JG et al. Outcomes in mild acute ischemic stroke treated with intravenous thrombolysis: a retrospective analysis of the Get With the Guidelines-Stroke registry. JAMA Neurol 2015; 72: $423-31$

\title{
Thrombektomie beim embolischen Hirninfarkt
}

\author{
Mehrere Studien, darunter die SWIFT PRIME-Studie, zeigen, dass eine \\ Thrombektomie bei intrakraniellen Gefäßverschlüssen der alleinigen \\ systemischen Lysetherapie eindeutig überlegen ist.
}

$\mathrm{n}$ den letzten Monaten sind mehrere große Studien publiziert worden, die alle dasselbe Ergebnis zeigen: die mechanische Thrombektomie ist der alleinigen systemischen Thrombolyse eindeutig überlegen.

Beispielhaft soll hier die SWIFT PRIME Studie referiert werden, die den Einsatz der mechanischen Thrombektomie mit dem Solitaire-Katheter untersuchte. Die Patienten mussten einen intrakraniellen Verschluss der A. carotis interna und/oder des M1-Segments aufweisen und erhielten zusätzlich eine systemische Thrombolyse. Die Patienten durften keinen großen Infarktkern und keine ausgedehnten Infarktfrühzeichen aufweisen. Die Intervention wurde innerhalb von 6 Stunden begonnen.

Ausgeschlossen wurden Patienten mit Verschluss der extrakraniellen A. carotis interna oder mit einer Kontraindikation für eine systemische Thrombolyse. Primärer Endpunkt war die funktionelle Unabhängigkeit gemessen auf der modifizierten Rankin Scale (mRS) nach 90 Tagen. Die Studie wurde vorzeitig nach Einschluss von je 98 Patienten in den beiden Gruppen (d.h. mit und ohne mechanische Thrombektomie) beendet.

Nach Thrombektomie lag die Rate einer weitgehenden oder vollständigen Reperfusion bei $88 \%$. Der primäre Endpunkt (mRS $0-2$ ) wurde von $60 \%$ nach

\section{Hier steht eine Anzeige.}

\author{
亚 Springer
}

